https://doi.org/10.48009/2_iis_2007_437-442

\title{
MEASURING THE IMPACT OF ENTERPRISE ARCHITECTURE
}

\author{
Sheila Cane, The MITRE Corporation, Sheila@mitre.org \\ Richard McCarthy, Quinnipiac University, Richard.mccarthy@quinnipiac.edu
}

\begin{abstract}
Enterprise architecture frameworks provide a basis to systematically document and manage the information technology assets of an organization. Numerous frameworks have emerged to support large scale organizations and government entities but to date there has been no empirical support to determine if they meet the needs of their users. We present a research model to enable empirical testing of the extent to which enterprise architecture frameworks support the task needs of their users. This model extends the existing task-technology fit research by combining the benefits of organizational technology fit with individual task needs. Further, it extends enterprise architecture research by providing a theoretical model to assess the fit of enterprise architecture frameworks.
\end{abstract}

Keywords: Task-technology fit, enterprise architecture, information systems strategy

\section{INTRODUCTION}

Enterprise architecture (EA) frameworks have been defined by Zachman [12], Spewak [10], and the United States Department of Defense [2] among others. These frameworks are used to enhance an organization's ability to promote transformational organizational change to model organizational processes, identify candidate process for change, and identify computer functions and systems to be used to implement those changes [7]. Enterprise archgitecture is a relatively new concept that has exploded onto the IT community and is emerging as a significant discipline within many large IT organizations. Several commercial entities have developed software tools for the enterprise architect's use. While the tools may implement the frameworks, it is not clear whether these frameworks (and by implication, the tools), meet the needs of their users to identify areas for change, help to specify computer systems to implement those changes, and whether the changes actually result in organizational performance improvement. This research applies the tasktechnology fit concepts found in Goodhue [3], Goodhue and Thompson [4], Dishaw and Strong [1], Zigurs and Buckland [13], and Khazanchi [6] to develop a model to determine if enterprise architecture frameworks meet the task needs of their users to facilitate transformational organizational change.

\section{LITERATURE REVIEW}

\section{Task Technology Fit}

Goodhue [3] theorized that an information system having task-technology fit would lead to increased individual performance. Goodhue and Thompson [4] developed the technology-to-performance chain model, shown in Figure 1, to investigate how technology can affect an individual's performance. Goodhue and Thompson [4] operationalized tasktechnology fit as user evaluation and specified the task-technology fit construct as consisting of the following variables: data quality, locatability of data, authorization to access data, data compatibility between systems, training and ease of use, production timeliness, systems reliability, and information systems relationship with users. Goodhue and Thompson [4] found strong support that the datarelated factors of task-technology fit predict perceived performance impacts, and that both utilization and task-technology fit must be included to predict performance.

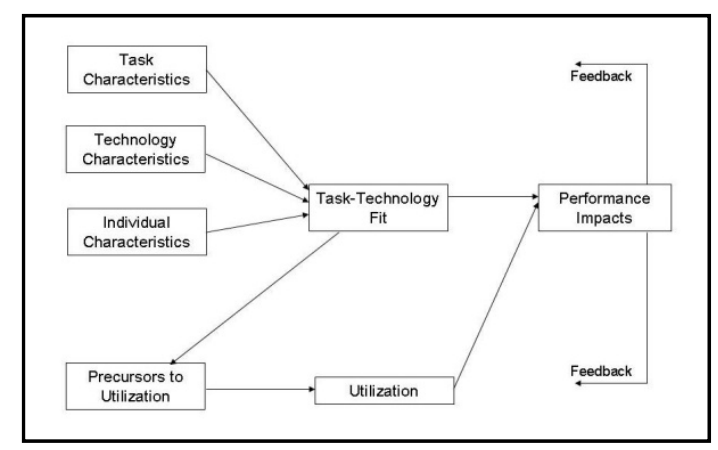

Figure 1 Goodhue and Thompson's [4] Technology-to-Performance Chain Model

Where Goodhue and Thompson [4] operationalized task-technology fit as user evaluation, Dishaw and Strong [1] operationalized task-technology fit in the context of software maintenance tools using the statistical method of computing fit as the interaction of task needs and technology functions. Further differing from Goodhue and Thompson's [4] list of task-technology fit factors, Dishaw and Strong's [1] 
method depended on the ability to directly specify the technology functions and task needs to be measured. Dishaw and Strong [1] used survey questions from the software maintenance literature (Vessey, 1986; Henderson \& Cooprider, 1990; in Dishaw \& Strong, 1998) to operationalize task-technology fit as the dimensions of production fit and coordination fit, where production fit is the interaction of software maintenance tool production functions with software maintenance tasks, and coordination fit is the interaction of software maintenance coordination tools functions with software maintenance coordination tasks.

Zigurs and Buckland [13] operationalized tasktechnology fit in the context of group support systems as task and technology alignments, testing for the effects of task-technology fit on group performance. Zigurs and Buckland's [13] task dimension was based upon task complexity, and considered five task categories: simple, problem, decision, judgment, and fuzzy. Zigurs and Buckland [13] derive unique research models from aligning distinct task environments with ideal technologies and testing for group performance effects which are defined for specific tasks.

Closely related to task-technology fit, Khazanchi [6] examined the benefits of organizational-technology fit in an exploratory research study whose objective was to identify candidate contingent factors for evaluation of information technology in terms of its "fit" or "appropriateness" for adoption and its potential benefit to an organization. Khazanchi [6] draws on Van de Ven and Drazen's (1985; in Khazanchi, 2005) concept of fit in contingency theory, where fit is described as 'congruence, internal consistency of multiple contingencies, structural and performance constructs.' Using Van de Ven and Drazen's (1985; in Khazanchi, 2005) systems approach, fit is defined as 'a feasible set of equally effective, internally consistent patterns of organization, context and structure,' 'organizational design can only advance if we address, in simultaneous manner, the many contingencies, structural alternatives, and performance criteria inherent to organizational life,' and that 'organizational performance is a function of match, congruence, intersection, or union of two or more factors.' Applying Van de Ven and Drazen's (1985; in Khazanchi, 2005) systems view of fit as matching, Khazanchi [6] identifies technology appropriateness as addressing the readiness of a firm for new information technology, and organizationaltechnology fit as addressing the potential impact of the new technology on organizational performance.
Khazanchi [6] operationalized performance by using subject matter expert (SME) assessment of relative benefits realized through the adoption and integration of Electronic Data Interchange (EDI). Relative benefits were assessed using a 5-point Likert-type scale ranging from 1: 'substantially deteriorated (or decreased)' to 3: 'no change' to 5: 'substantially improved (or increased). The potential EDI benefits identified by Khazanchi [6] are listed in Table 1 .

\section{TABLE 1}

Khazanchi's [6] Potential EDI Benefits

Quality of Information

Relationship with Trading Partners

Customer Service

Ability to Compete

Operational Efficiency

Cash Flows

Transaction Costs (reverse coded)

Inventory Levels (reverse coded)

\section{Enterprise Architecture and Strategic Change}

Luftman et al. [7] identify changes that drive business transformation: the breakdown of distinction between large and small businesses due to strategic alliances and partnering; the need for flexible and agile business structures due to globalization; a work force shifting from blue and white collar to knowledge workers; manager's role changing from controlling to coaching and guiding; the need for new competitive strategies based upon cost effective shifting from mass production to mass customization; and an increasing rate of technology innovation and introduction.

Information technology can provide strategic value to businesses as they cope with these changes by supporting administrative infrastructure, key business and operational processes, and can be used to "reduce costs, enhance product and service quality, improve customer service, integrate supplier and customer operations, and enable organizational learning [7].” Luftman et al. [7] further states, "the alignment or fit of information technology with the business is fundamental to the notion of transformation," and organizations should look at how information technology can enhance the organization's competitive performance, rather than how it can solve a particular operating problem.

Rico [9] defines enterprise architecture as "a comprehensive framework or taxonomy of systems 
analysis models for aligning organizational strategy with information technology". The US Government defines enterprise architecture "as a strategic information asset base, which defines the business, the information necessary to run the business, the technologies necessary to support the business operations, and the transitional processes necessary for implementing new technologies in response to the changing needs of the business" [8]. Enterprise architecture is used to define an organization's scope, business model, systems model, technology model, and components [9]. Enterprise architecture holistically explains how the enterprise's information technology elements work together, including systems, people, processes, and organizations [8].

Organizations need methods to help them cope with increased rates of change, and to manage their organizational and technology strategy [11]. Veasey [11] describes how enterprise architecture can be used to create a model of the organization that will facilitate organizational change and manage complexity. Veasey designed an architecture framework with which one can describe an enterprise's current state and future state, and be used to decide upon and communicate the organizational change strategy. Veasey's enterprise architecture framework addresses the following components: stakeholders, capabilities, culture, competencies, process, organization and technology. Veasey recommends that stakeholders be analyzed according to how their differing demands should be balanced, and views capabilities as having five elements: processes, which deliver capabilities, and are supported by culture, technology and organization. Competencies are individual's attributes that when combined create enterprise competence. Veasey [11] further states that process is a coordination of activity and should be managed separately as workflow, and distinguished from data and information processing.

Veasey [11] states that enterprise architecture can be implemented using program management techniques to implement strategy. To this aim, Veasey [11] provides an architecture development process and a distributed hierarchical implementation strategy. Veasey's [11] process includes identifying stakeholders, identifying the current capabilities and the required capabilities to create the vision, identifying the elements needed to meet the capabilities, creating the strategy by defining strategic programs, and by defining and implementing projects. Veasey's [11] hierarchy, called business federation design (BFD) is used to create parent/child enterprises built around implementation responsibilities. Parent enterprise capabilities include enterprise management, stakeholder valued capabilities, and parenting capabilities. Child enterprises are either responsible for providing capabilities or for parenting another sub-enterprise. Veasey [11] suggests using the categories from the framework as template headings for capturing information about the enterprise architecture. Veasey concludes that by using this framework and process, enterprise architects can manage change, satisfy stakeholders, understand key implementation issues, sustain change momentum, build holistic capabilities, and create a learning environment.

Technology transformation seeks to implement business strategy using information technology but without the constraints of the current organizational design. Methods for technology transformation include technology forecasting and architectural planning [5].

Luftman et al. [7] developed a strategic alignment framework to apply to Henderson and Venkatraman's [5] Strategic Alignment model. Luftman et al. [7] identify the purpose of their framework as "to apply knowledge and technology to accelerate the transformation of companies into successful organizations that will be sustainable in the future." Luftman et al.'s [7] strategic alignment framework attempts to harmonize "business strategy, information technology strategy, organizational infrastructure and processes, and I/T infrastructure and processes" ... "to build an organizational structure and set of business processes that reflect the interdependence of enterprise strategy and information technology capabilities" and "can significantly affect the competitiveness and efficiency of the business.” Luftman et al.’s [7] methods for transformation include: 1) linking the strategic direction of the business to specific leveraging technologies by creating an information technology strategy, an information technology plan, and a set of initiatives, 2) using total quality methods and business re-engineering to transform the organization, 3) creating systems architectures with detailed information on application development, workstations, networking and management, and 4) identifying the dominant strategic perspective from the Strategic Alignment Model and use the methods defined for that perspective as an implementation approach.

Zachman [12], when presenting his seminal work in architecture frameworks, acknowledged the need for a strategic planning methodology that links business strategy to strategic planning, while not pursuing 
strategic planning per se. The Zachman framework [12], developed from the study of classical building architecture processes, includes a set of architectural representations that can represent "different perspectives of different participants" and descriptions of same product in different ways for different purposes. Zachman's [12] architecture representations include three description types: functional description, or process model; material description, or data model, where the data model is viewed similarly to a bill-of-materials; and the location description, or the network model, which focuses on the flows between components. Zachman's [12] framework include several perspectives: scope, or ballpark perspective, which include things that matter to a business; the business model, or owner's perspective, which includes business entity relationships; the information system, or designer's perspective, which includes the system entity relationships; the technology model, or builder's perspective, which is the physical implementation or data design; and the detailed description, or out-of-context perspective, which specifies the data fields and relationships. Zachman [12] suggests that there are reasons to develop architectural products for each of the descriptions for each perspective, and identifies the risks of not developing any one as future research.

Morganwalp and Sage [8] explain how an enterprise architecture should be considered a complex system of systems because enterprise architectures have the following five characteristics: operational independence of the individual systems, managerial independence of the individual systems, geographic distribution, emergent behavior, and evolutionary and adaptive development. As enterprises grow, the relationships between the organizations, processes, people, and information technology systems and subsystems can become quite challenging and complex, and an enterprise architecture framework should be able to look at the systems, subsystems and their relationships as a whole [8].

Rico [9] identifies principles for successful enterprise architecture. Foremost, the organization must clearly understand the enterprise architecture's objectives, which can include, for example, compliance with government regulations or to control information technology investment. The organization should also be able to articulate the expected benefits of the architecture, and have a clearly designed strategy. Rico [9] identifies the following principles for success: clear vision; early, measurable objectives; incremental development of small, well-defined scope implemented by highly experienced teams; managed using project management techniques including the use of earned value management, quality management and return on investment to track progress; application of top-down systems analysis; creation of style guides and standards; and thinking outside the box for tool selection.

\section{Enterprise Architecture Success Measures}

Morganwalp and Sage [8] identify three approaches for evaluating the effectiveness of an enterprise architecture:

1.building a system based on the EA and measuring its performance

2. developing an executable model of a proposed architecture and experimentally determining the expected performance of the architecture before it is actually built

3. seeking expert opinion regarding the likely performance of a given architecture

Through a review of the literature, Morganwalp and Sage [8] identify methods for assessing the qualitative value of an enterprise architecture, including the 'key metrics' defined by the META Group (1998; in Morganwalp \& Sage, 2004) of organizational coverage, technical architecture process performance, and component content utility; simple effectiveness measures such as reduced number of vendors of the same technology over time, reduced version released diversity, reduced number of aged internal standards, and reduced review process cycle time (Stewart, 2001: in Morganwalp \& Sage, 2004); user evaluation of effectiveness (Treasury, 2000; in Morganwalp \& Sage, 2004); value (Boster, Liu \& Thomas, 2000; in Morganwalp \& Sage, 2004); and the quality measures of financial efficiency, business effectiveness and architecture process (Buchanan, 2001; in Morganwalp \& Sage, 2004).

Rico [9] identifies ways to measure return on investment of enterprise architecture, and states that impact can be measured using the measures of cost, benefits, benefit to cost ratio, return on investment, net present value, and breakeven point, where costs and benefits are the basis for calculation of the other measures. Costs include those costs associated with training, software tools, time spent creating architecture models, verification and validation, and compliance/maturity assessment. Benefits are monetized measures of increased operational efficiency, reduced operational costs, reduced personnel numbers, increased customer satisfaction, and the consolidation of legacy systems [9]. Rico [9] identifies as the monetization of benefits as necessary 
to calculate return on investment, but difficult to accomplish for enterprise architecture development. Rico [9] also suggests that organizations quantitatively operationalize the following as a core set of metrics influencing the return on investment for enterprise architecture: people, time, budgets, customers, throughput, volume, bandwidth, computers, and maintenance.

\section{RESEARCH QUESTIONS}

The concept of task-technology fit is important one; an understanding of how to measure the fit between technologies and task needs can help identify those technologies able to enhance individual and organizational performance, as well as to prevent the unnecessary purchase of software and systems. A review of the literature shows that no single model of task-technology fit has emerged.

Enterprise architecture has been defined as a set of methods and software tools that can be used to facilitate organizational transformation. A review of the enterprise architecture literature shows that although there are some proposed ways of measuring the successful implementation of enterprise architecture in terms of financial, operational and customer service measures, these measures are made after the investment in implementing an enterprise architecture program. There has been little empirical research on the effectiveness of enterprise architecture frameworks, or by implication the software tools that are used to implement these frameworks for the purposes of managing technology for strategic organizational change.

This research proposes the application of the tasktechnology fit models of Goodhue [3], Goodhue and Thompson [4], Dishaw and Strong [1] and Zigurs and Buckland [13], and Khazanchi's [6] organizational benefits to address the following question: Do enterprise architecture frameworks meet the needs of their users to manage technology and facilitate transformational organizational change?

\section{RESEARCH MODEL}

To address the differences in approach taken by various researchers in task-technology fit research, this research proposes a combined model, selecting constructs relevant to measuring the task-technology fit of enterprise architecture tools from several of the extant research models. The proposed research model, shown in Figure 2, is based upon Goodhue's [3] task-technology fit model, is derived from the above literature review, and combines relevant constructs from the task-technology fit models by addressing user perception of fit as described by Goodhue [3], the need to define technology functions, as articulated by Dishaw and Strong [1], task complexity as described by Zigurs and Buckland [13], utilization and performance as described by Goodhue, [3] and organizational benefits as described by Khazanchi [6].

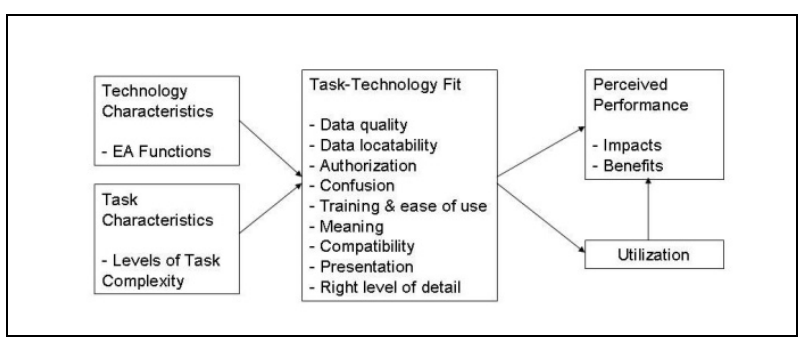

Figure 2 Proposed Research Model

Goodhue [3] pre-defined a data focused set of tasktechnology fit dimensions for user evaluation of tasktechnology fit. The proposed research model includes those dimensions relevant to enterprise architecture. Dishaw and Strong [1] viewed functions and tasks as important in determining tasktechnology fit. Following Dishaw and Strong's [1] concepts, the relevant functions of EA will be derived from the literature and tested as dimensions of the technology characteristics function. Zigurs and Buckland's [13] complexity levels will be used as dimensions of task characteristics. Perceived performance impacts and benefits will include both individual performance as identified by Goodhue [3] and organizational benefits as defined by Khazanchi [6]. Goodhue and Thompson's [4] technology to performance chain model does not change; however some of the variables used to measure each construct are modified.

\section{DISCUSSION}

This research model continues the task-technology fit research by integrating several different perspectives to examine the dimensions within the tasktechnology fit constructs and continue the evolution of the task-technology fit theoretical model. The research extends past efforts to find a task-technology fit model that enables organizations to evaluate technology from a task-need's perspectives prior to a software purchase. It provides a theoretical basis for empirical testing of enterprise architecture frameworks. This model extends the existing tasktechnology fit research by examining the benefits of organizational technology fit with individual task needs. Finally, it evaluates whether enterprise 
architecture frameworks meet the task-needs of their users, and whether as a result, enterprise architecture frameworks can help to facilitate transformational organizational change.

\section{SIGNIFICANCE OF THE RESEARCH}

While Enterprise Architecture tools and techniques have been widely adopted, there have been no empirical studies done on its effectiveness and the technical community is starting to question it's effectiveness in achieving organizational change The task technology fit model has not converged on a single method that can be used to evaluate technology prior to or post implementation. If we outline all the possible variables defining enterprise architecture functions, task complexity, user evaluation of fit, and user evaluation of performance of EA, we can identify which factors are key to perceived fit and performance and successful implementation of EA for facilitating organizational change. The secondary benefit is to further theoretical advances in understanding and describing the task-technology fit model.

\section{REFERENCES}

1. Dishaw, M.T., \& Strong, D.M. (1998), Supporting Software Maintenance with Software Engineering Tools: A Computed TaskTechnology Fit Analysis, The Journal of Systems and Software, 44(2), 107-120.

2. DoD Architecture Framework Working Group. DoD Architecture Framework Version 1.0, Volume I: Definitions and Guidelines, 2004, Downloaded 5/18/06 from http://www.defenselink.mil/nii/doc/DoDAF_v1_ Volume_I.pdf.

3. Goodhue, D.L. (1995). Understanding User Evaluations of Information Systems, Management Science, 41(12), 1827-1844.

4. Goodhue, D. L., \& Thompson, R. L. (1995), Task-Technology Fit and Individual Performance, MIS Quarterly, 19(2), 213-236.

5. Henderson, J.C., \& Venkatraman, N. (1999), Strategic Alignment: Leveraging Information Technology for Transforming Organizations, IBM Systems Journal, 32(1), 472-484.

6. Khazanchi, D. (2005), Information Technology (IT) Appropriateness: The Contingency Theory of 'Fit' and IT Implementation in Small and Medium Enterprises, The Journal of Computer Information Systems, 45(3), 88-95.

7. Luftman, J.N., Lewis, P.R., \& Oldach, S.H. (1993), Transforming the Enterprise: The Alignment of Business and Information
Technology Strategies, IBM Systems Journal, 32(1), 198-221.

Morganwalp, J.M., \& Sage, A.P. "Enterprise Architecture Measures of

8. Effectiveness," Int. J. Technology, Policy and Management, (4:1), 2004, 81-94.

9. Rico, D.F. (2006), A Framework for Measuring ROI of Enterprise Architecture, Journal of Organizational and End User Computing, 18(2), i-xii.

10. Spewak, S.H., \& Hill, S.C. (1992), Enterprise Architecture Planning: Developing a Blueprint for Data, Applications, and Technology. John Wiley and Sons, Inc. New York, N.Y.

11. Veasey, P.W. (2001), Use of Enterprise Architectures in Managing Strategic Change, Business Process Management Journal, 7(5), 420-436.

12. Zachman, J.A. (1987), A Framework for Information Systems Architecture, IBM Systems Journal, 26(3), 276-292.

13. Zigurs, I., \& Buckland, B., K. (1998), A Theory of Task/Technology Fit and Group Support Systems Effectiveness, MIS Quarterly, 22(3), 313-334. 